\title{
Mesoscopic description of network-forming clusters of weakly charged colloids
}

\author{
A. Ciach, W.T. Góźdź \\ Institute of Physical Chemistry, Polish Academy of Sciences, 01-224 Warszawa, Poland
}

Received February 10, 2010, in final form March 18, 2010

Systems composed of spherical charged particles in solvents containing counterions and inducing effective short-range attraction are studied in the framework of mesoscopic field-theory. We limit ourselves to meanfield approximation (MF) and to weak ordering. We discuss properties of potentials consisting of strong shortrange attraction and weak long-range repulsion (SALR) in the context of formation of nonuniform distribution of particles on a mesoscopic length scale instead of macroscopic phase separation. In earlier work it was found that spherical, cylindrical and slab-like clusters of particles are formed, and for low enough temperatures the clusters form ordered, periodic bcc, hexagonal and lamellar phases. In addition, a gyroid phase was predicted in which two interwoven regular network-like clusters branching in triple junctions are formed. At properly rescaled density and temperature, the coexistence lines between different ordered phases were found to be universal in MF, with the exception of the gyroid phase. Here the phase diagram is determined for two choices of the SALR potential, one corresponding to a large range of the attractive part of the potential, and the other one to a very small range of attraction. We find that the region of stability of the gyroid phase very weakly depends on the form of the SALR potential within the approximate theory.

Key words: colloids, clusters, self-assembly, order-disorder phase transitions

PACS: $61.20 . G y, 64.60 . D e, 82.70 . D d$

\section{Introduction}

Biologically relevant macromolecules, nanoparticles or colloids are typically charged, and therefore repel each other. On the other hand, van der Waals and various solvent-mediated interactions lead to effective attraction between them. The range of attraction is usually shorter than the range of screened electrostatic repulsion. However, for weakly to moderately charged particles, the magnitude of the attraction is significantly larger than the magnitude of repulsion. The resulting potential is negative for short distances, and positive for larger distances and is known as short-range attraction long-range repulsion (SALR) potential [1-15]. Short separations of particles corresponding to the attraction are favorable, whereas the distances close to the maximum of repulsion lead to a significant increase in energy. Depending on the repulsion barrier and range, as well as on density $\rho$ and temperature $T$, the random distribution of particles and macroscopic phase separation can be less favorable than the formation of clusters of various shape and size. When the average distance between particles corresponds to strong repulsion, then it is energetically favorable to form clusters with particles strongly attracting each other, such that the separation between the clusters is larger than the range of repulsion. When separations between the particles forming the cluster are mainly within the range of attraction and the separation between clusters is larger than the range of repulsion, the energy takes low values. This effect may overcompensate the entropy loss associated with cluster formation. When the density increases such that the average separation between particles is too small to form spherical clusters with particles attracting each other, and with average separation between the clusters larger than the range of repulsion, then under the constraint of a given density, it is favorable to grow the cluster in one dimension, and keep the distance between elongated clusters larger than the range of repulsion. For still higher densities, slab-like clusters sufficiently far apart from each other may be created. Such scenario has been observed both in experiments and in simulations. In experimental systems the clusters have been 
directly seen by confocal microscopy [3, 7]; for an increasing density, first spherical, then elongated and finally network-like clusters were observed. In the case of globular protein solutions, cluster formation is a subject of intensive debate [3, 16]. Simulation [9, 11] and theoretical studies [17, 18] of particular examples of the SALR potential show that indeed spherical, cylindrical and slab-like clusters are formed for an increasing density. In addition, for some range of particle density, lower than the density of crystallization, the clusters form ordered, periodic structures at low enough temperatures. The results of simulations and recent theoretical prediction [17, 18] indicate that the sequence of ordered phases is the same regardless of the detailed form of the SALR potential. In particular, small [11] and very large [9] clusters are spherical, cylindrical and slab-like in shape for similar volume fractions of particles. Mean-field results [17] indicate that for a suitably rescaled temperature, the phase diagram is universal when weak ordering of particles occurs, i.e., in rescaled variables the phase-coexistence lines for different forms of the SALR potential collapse onto master curves. There is one exception, however. Between the stability regions of the hexagonal phase of cylinders and lamellar phase of slabs, a thermodynamically stable gyroid phase was found [17], but its stability region is not universal, i.e., it may be different for different forms of the effective interactions. In this phase particles form two interwoven networks of clusters which branch in triple junctions. This phase may be related to the branched network of clusters observed experimentally and in simulations [6, 7, 14]. The prediction of stability of the gyroid phase is based on mean-field approximation to a mesoscopic field theory, and the results were obtained under assumption of weak ordering (i.e., strong fluctuations of particles around their average positions, leading to smooth concentration profiles with small amplitudes). Hence, further evidence is required to confirm that such a peculiar regular 'gel' can be a thermodynamically stable phase.

In this work we address an open issue how the stability region of this gyroid phase on the phase diagram depends on the shape of the SALR potential, and consequently on the size of the equilibrium clusters at strong dilution. To this end, we consider two forms of the SALR potential, one leading to very large and the other one to very small clusters, and determine the phase diagrams for these two versions of the SALR potential using the method developed in [17].

In section 2 we introduce a particular form of the SALR potential, consisting of two Yukawa terms, and discuss the range of parameters corresponding to phase separation and to clustering. In section 3 we briefly review the version of the statistical field theory introduced in [17]. This approach is suitable for a description of collective phenomena leading to ordering on the mesoscopic length scale. In section 4 we describe the calculations of the phase diagram in the simplest mean-field approximation in the case of weak ordering, and discuss our results.

\section{The interaction potential and its properties}

We restrict our attention to colloids, nanoparticles or globular macromolecules that can be modeled as spherical hard cores with diameter $\sigma$, and with additional interactions present for distances $r>\sigma$. We limit ourselves to the interaction potential that has the form

$$
u(r)=u^{\mathrm{SR}}(r)+u^{\mathrm{LR}}(r)
$$

with

$$
u^{\mathrm{SR}}(r)=\left\{\begin{array}{lll}
0 & \text { if } & r>1 \\
\infty & \text { if } & 0<r<1
\end{array}\right.
$$

where $r$ is in $\sigma$ units. The long-range part $u^{\mathrm{LR}}(r)$ is defined for $r>1$, and for such separations we assume that $u^{\mathrm{LR}}(r)$ has the form

$$
v(r)=-\frac{\mathcal{A}_{1}}{r} \mathrm{e}^{-z_{1} r}+\frac{\mathcal{A}_{2}}{r} \mathrm{e}^{-z_{2} r},
$$

where $z_{i}$ is the inverse range in $\sigma^{-1}$ units. We consider the SR and the LR parts separately and treat the case with $u^{\mathrm{LR}}(r)=0$ as a reference system. In microscopic theory we need an extension 
of $u^{\mathrm{LR}}(r)$ for $r<1$ for calculational reasons. The contribution to the energy of the system coming from overlapping cores should be avoided, and therefore we assume

$$
u^{\mathrm{LR}}(r)=\left\{\begin{array}{lll}
v(r) & \text { if } & r \geqslant 1, \\
0 & \text { if } & r<1 .
\end{array}\right.
$$

Such an extension is not unique, and other choices made in [19 22] in different context lead to more accurate results. The simplest possibility of extension of the long-range part of the potential for $r<1$ would be to assume the form (3) for all $r \geqslant 0$. In this case analytical results can be easily obtained. However, the self-energy would be included, and moreover, there would be no information on the length scale set by the hard cores. We shall compare the structure factor obtained within the simplest Gaussian approximation for $u^{\mathrm{LR}}(r)=v(r)$ and for $u^{\mathrm{LR}}(r)$ given by (4) in the next section; the form (4) leads to the structure factor that qualitatively agrees with experiments and simulations, whereas for $u^{\mathrm{LR}}(r)=v(r)$ the second peak of the structure factor, associated with particle-particle correlations within the clusters is missing.

In the mesoscopic theory [17, 18] the key role is played not by the potential alone, but rather by the potential multiplied by the microscopic correlation function, $g(r)$, calculated for the frozen mesoscopic density distribution. The form of $g(r) u^{\mathrm{LR}}(r)$ is given by (4) for any extension of $u^{\mathrm{LR}}(r)$ for $r>1$ for the simplest approximation $g(r) \approx \theta(r-1)$.

We focus on the case of strong short-range attraction and weak long-range repulsion, and assume $z_{1}>z_{2}$, and $\mathcal{A}_{1}>\mathcal{A}_{2}$. In Fourier representation (4) takes the form

$$
\tilde{u}^{\mathrm{LR}}(k)=4 \pi\left[\frac{\mathcal{A}_{2} \mathrm{e}^{-z_{2}}}{z_{2}^{2}+k^{2}}\left(z_{2} \frac{\sin k}{k}+\cos k\right)-\frac{\mathcal{A}_{1} \mathrm{e}^{-z_{1}}}{z_{1}^{2}+k^{2}}\left(z_{1} \frac{\sin k}{k}+\cos k\right)\right] .
$$

We use the notation $\tilde{f}(k)$ for the Fourier transform of the function $f(r)$. Note that the energy associated with a plane wave of concentration, $\rho^{*}(z)=\Phi \cos (k z)+\bar{\rho}^{*}$, is equal to $V \Phi^{2} \tilde{u}^{\mathrm{LR}}(k) / 4$, where $V$ is the volume of the system and we introduced dimensionless density

$$
\rho^{*}=\rho \sigma^{3}
$$

and denoted the space-averaged density by $\bar{\rho}^{*}$. When $\tilde{u}^{\mathrm{LR}}(k)<0$, a concentration wave with the wavelength $2 \pi / k$ leads to a decrease of energy compared to homogeneous distribution of particles. The minimum of $\tilde{u}^{\mathrm{LR}}(k)$ at $k=k_{\mathrm{b}}$ gives the most probable wavelength $2 \pi / k_{\mathrm{b}}$ of the plane concentration wave, where layers with excess particle density of width $\pi / k_{\mathrm{b}}$ are followed by regions of depleted particle density of the same width. Superpositions of plane waves in different directions may lead to nonuniform distribution of particles of different symmetries. The energy associated with the most probable wave of concentration, $\tilde{u}^{\mathrm{LR}}\left(k_{\mathrm{b}}\right)$ is a convenient energy unit, and we introduce dimensionless temperature $T^{*}$ by

$$
T^{*}=\frac{k_{\mathrm{B}} T}{\left|\tilde{u}^{\mathrm{LR}}\left(k_{\mathrm{b}}\right)\right|} .
$$

In [17] it was shown that in mean-field (MF) approximation, the spinodal lines are given by a universal curve in $\left(T^{*}, \rho^{*}\right)$ phase diagram, independently of the shape of the SALR potential. Thus, such units are appropriate for determination of the common features of different systems that form nonuniform structures on the mesoscopic length scale.

The gas-liquid phase separation may occur when $\tilde{u}^{\mathrm{LR}}(0)<0$, and the necessary condition for the phase separation is

$$
\frac{\mathcal{A}_{2}}{\mathcal{A}_{1}}<\mathrm{e}^{-\left(z_{1}-z_{2}\right)}\left(\frac{z_{1}+1}{z_{2}+1}\right)\left(\frac{z_{2}}{z_{1}}\right)^{2} .
$$

The behavior depends on whether $\tilde{u}^{\mathrm{LR}}(k)$ assumes a minimum or a maximum for $k=0$. We find

$$
\left.\frac{\mathrm{d}^{2} \tilde{u}^{\mathrm{LR}}(k)}{\mathrm{d} k^{2}}\right|_{k=0}=\frac{4 \pi}{3}\left[\frac{\mathcal{A}_{1} \mathrm{e}^{-z_{1}}\left(z_{1}^{3}+3 z_{1}^{2}+6 z_{1}+6\right)}{z_{1}^{4}}-\frac{\mathcal{A}_{2} \mathrm{e}^{-z_{2}}\left(z_{2}^{3}+3 z_{2}^{2}+6 z_{2}+6\right)}{z_{2}^{4}}\right] .
$$


For parameters $A_{i}, z_{i}$ corresponding to negative values of the above (maximum at $k=0$ ) we find periodic ordering, because the second extremum, for $k>0$, is a minimum. For $z_{1}>6$ and $z_{2} \approx 1$ this is typically the case if $\mathcal{A}_{2} / \mathcal{A}_{1}>10^{-4}$. Hence, even very weak repulsion suppresses phase separation for very short-range of attraction. For longer range of attraction we need a sufficiently strong repulsion to suppress phase separation in favor of clustering.

The parameters $A_{i}$ and $z_{i}$ may be state-dependent, and in different systems the dependence on temperature and density may be different. In particular, for charged colloids in the presence of counterions, $z_{2}$ is the inverse screening length which is a function of temperature and density of the form $z_{2} \propto \sqrt{\rho / T}$. Here we are not interested in any particular system, but rather try to determine the shape of the phase diagram in the universal variables. Note that when the potential depends on temperature and density, the same holds for the energy unit $\left|\tilde{u}^{\mathrm{LR}}\left(k_{\mathrm{b}}\right)\right|$.

We choose two sets of parameters for the potential given by (3).

$$
\begin{aligned}
& \mathcal{A}_{1}=1, \quad \mathcal{A}_{2}=0.2, \quad z_{1}=1, \quad z_{2}=0.5, \\
& \mathcal{A}_{1}=1, \quad \mathcal{A}_{2}=0.05, \quad z_{1}=3, \quad z_{2}=0.5 \text {. }
\end{aligned}
$$

The shape of the potential in the cases I and II is quite different. In the case I large clusters are formed, because $k_{\mathrm{b}} \approx 0.6088$ whereas in the case II the clusters are rather small, because $k_{\mathrm{b}} \approx 1.7926$.

\section{Field theory}

Consider nonuniform density distributions $\rho^{*}(\mathbf{x})$. The probability that the density has the given form $\rho^{*}(\mathbf{x})$ in the mesoscopic description is given by [17]

$$
p\left[\rho^{*}(\mathbf{x})\right]=\Xi^{-1} \mathrm{e}^{-\beta \Omega^{\mathrm{MF}}\left[\rho^{*}(\mathbf{x})\right]},
$$

where $\beta=1 /\left(k_{\mathrm{B}} T\right), k_{\mathrm{B}}$ is the Boltzmann constant, and $\Omega^{\mathrm{MF}}\left[\rho^{*}\right]$ is the grand-thermodynamic potential in the system with the density constrained to have the given nonuniform form $\rho^{*}(\mathbf{x})$,

$$
\beta \Omega^{\mathrm{MF}}\left[\rho^{*}\right]=\frac{1}{2} \int_{\mathbf{x}} \rho^{*}(\mathbf{x}) \beta u^{\mathrm{LR}}\left(\left|\mathbf{x}-\mathbf{x}^{\prime}\right|\right) \rho^{*}\left(\mathbf{x}^{\prime}\right)+\int_{\mathbf{x}}\left(\beta f_{\mathrm{h}}\left[\rho^{*} \mid \mathbf{x}\right)-\mu^{*} \rho^{*}(\mathbf{x})\right)
$$

where $\mu^{*}=\beta \mu / \sigma^{3}$, and we use the notation $\int_{\mathbf{x}} \equiv \int \mathrm{d} \mathbf{x} \cdot f_{\mathrm{h}}\left[\rho^{*} \mid \mathbf{x}\right)$ is the free-energy density of the reference system (with $u^{\mathrm{LR}}=0$ ) at $\mathbf{x}$. In general, $f_{\mathrm{h}}\left[\rho^{*} \mid \mathbf{x}\right)$ is a functional of $\rho^{*}(\mathbf{x})$. Here we limit ourselves to the local-density approximation, where the free-energy density at the space position $\mathbf{x}$ is a function of the local density at $\mathbf{x}$

$$
f_{\mathrm{h}}\left[\rho^{*} \mid \mathbf{x}\right)=f_{\mathrm{h}}\left(\rho^{*}(\mathbf{x})\right)
$$

We adopt the PY approximation for the free energy of hard spheres at density $\rho^{*}$,

$$
\beta f_{\mathrm{h}}\left(\rho^{*}\right)=\rho^{*} \ln \left(\rho^{*}\right)-\rho^{*}+\rho^{*}\left[\frac{3 s(2-s)}{2(1-s)^{2}}-\ln (1-s)\right],
$$

where $s=\rho^{*} \pi / 6$. Note that in this theory the form of $\Omega^{\mathrm{MF}}\left[\rho^{*}(\mathbf{x})\right]$ depends on the choice for the extension of $u^{\mathrm{LR}}(r)$ for $r<1$ (see the first term in equation (12)). Let us first consider $\beta \Omega^{\mathrm{MF}}$ for uniform states $\rho^{*}=$ const.

$$
\beta \Omega^{\mathrm{MF}}\left(\rho^{*}\right) / V=-\beta^{*} p^{*}=\frac{\rho^{* 2}}{2} \beta^{*} \tilde{u}^{* \mathrm{LR}}(0)+\beta f_{\mathrm{h}}\left(\rho^{*}\right)-\mu^{*} \rho^{*},
$$

where we used the thermodynamic relation $\Omega=-p V$, introduced $\tilde{u}^{* \mathrm{LR}}(k)=\tilde{u}^{\mathrm{LR}}(k) /\left|\tilde{u}^{\mathrm{LR}}\left(k_{\mathrm{b}}\right)\right|$, $\beta^{*}=1 / T^{*}$, and $p^{*}=p /\left|\tilde{v}\left(k_{\mathrm{b}}\right)\right|$. 

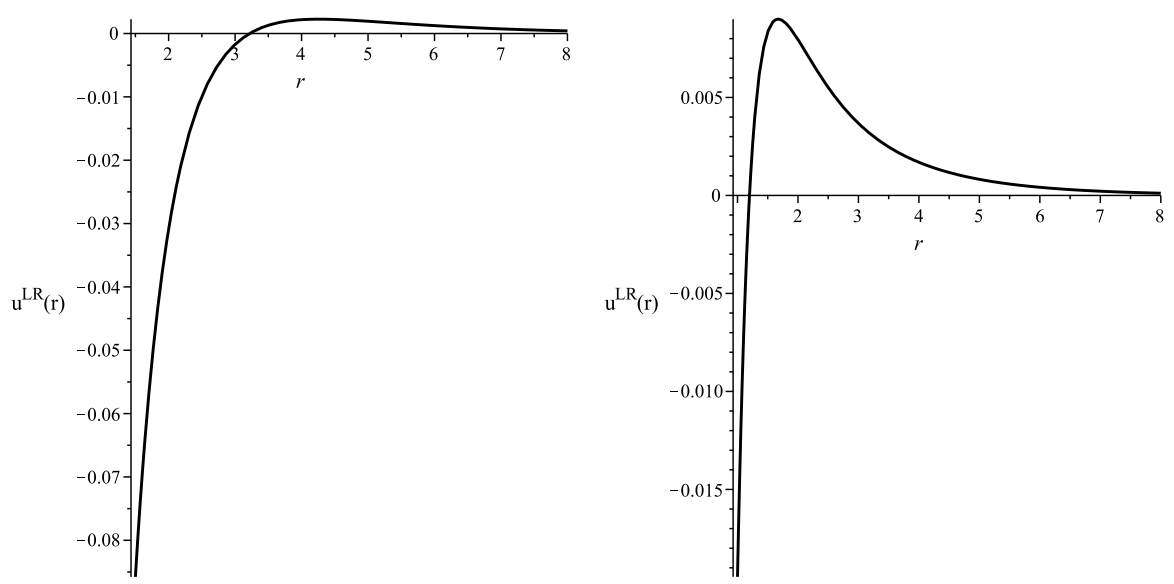

Figure 1. The LR part of the interaction potential, $u^{\mathrm{LR}}(r)$ given by (4), for two choices of parameters. Left: $\mathcal{A}_{1}=1, \mathcal{A}_{2}=0.2, z_{1}=1, z_{2}=0.5$. Right: $\mathcal{A}_{1}=1, \mathcal{A}_{2}=0.05, z_{1}=3$, $z_{2}=0.5$.
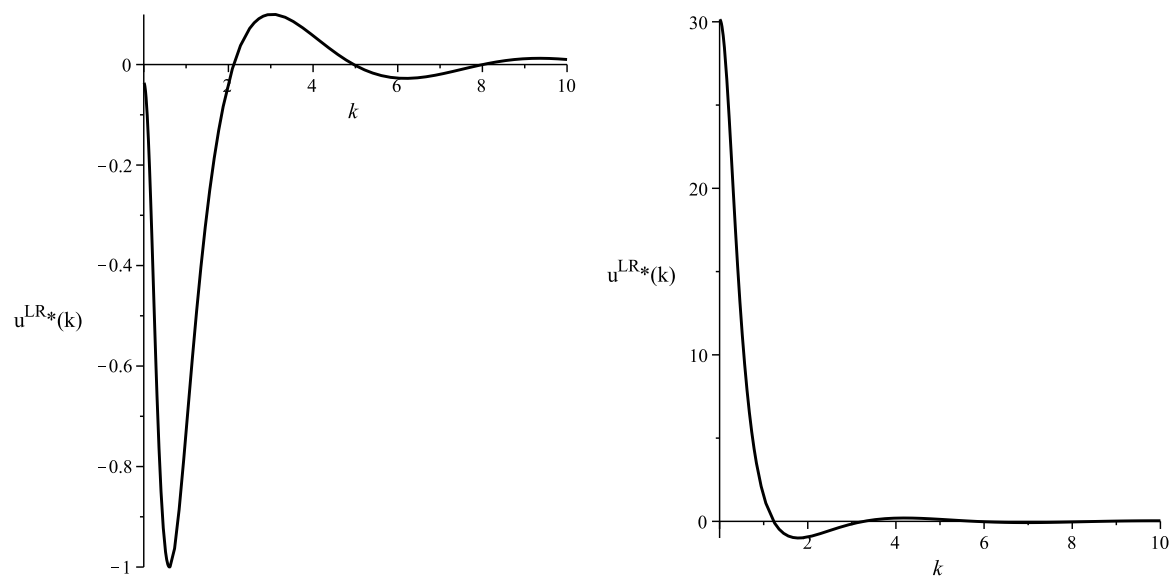

Figure 2. Fourier transform of the LR part of the interaction potential, $\tilde{u}^{\mathrm{LR}}(k)$ given by (5), for two choices of parameters. Left: $\mathcal{A}_{1}=1, \mathcal{A}_{2}=0.2, z_{1}=1, z_{2}=0.5$. Right: $\mathcal{A}_{1}=1, \mathcal{A}_{2}=0.05$, $z_{1}=3, z_{2}=0.5$.

Minimum condition of $\Omega^{\mathrm{MF}}\left(\rho^{*}\right)$ :

$$
\frac{\partial \Omega^{\mathrm{MF}}}{\partial \rho^{*}}=0
$$

has the explicit form

$$
\rho^{*} \beta \tilde{u}^{\mathrm{LR}}(0)+\frac{\mathrm{d} \beta f_{\mathrm{h}}\left(\rho^{*}\right)}{\mathrm{d} \rho^{*}}=\mu^{*}
$$

and

$$
\tilde{u}^{\mathrm{LR}}(0)=\int_{\mathbf{x}} u^{\mathrm{LR}}(x)=4 \pi\left[\frac{\mathcal{A}_{2} \mathrm{e}^{-z_{2}}}{z_{2}^{2}}\left(z_{2}+1\right)-\frac{\mathcal{A}_{1} \mathrm{e}^{-z_{1}}}{z_{1}^{2}}\left(z_{1}+1\right)\right] .
$$

Densities that satisfy the above for a given $\mu^{*}$ and $T^{*}$ correspond to the most probable density of 
the uniform system and are denoted by $\rho_{0}^{*}$. The explicit form of (16) is

$$
\mu^{*}=\rho_{0}^{*} \beta^{*} \tilde{u}^{* \operatorname{LR}}(0)+\ln \left(\rho_{0}^{*}\right)-\ln \left(1-s_{0}\right)+\frac{14 s_{0}-13 s_{0}^{2}+5 s_{0}^{3}}{2\left(1-s_{0}\right)^{3}} .
$$

The correlation function for mesoscopic density in the Gaussian approximation is just given by

$$
\tilde{G}(k)=\frac{1}{\tilde{C}(k)}=\frac{\rho_{0}^{*}(1-s)^{4}}{\beta^{*} \tilde{u}^{* \operatorname{LR}}(k) \rho_{0}^{*}(1-s)^{4}+(1+2 s)^{2}},
$$

where

$$
\tilde{C}(k)=\frac{\delta^{2} \beta \Omega^{\mathrm{MF}}}{\delta \tilde{\rho}^{*}(k) \delta \tilde{\rho}^{*}(-k)}=\beta^{*} \tilde{u}^{* \mathrm{LR}}(k)+\frac{\mathrm{d}^{2} \beta f_{\mathrm{h}}\left(\rho^{*}\right)}{\mathrm{d} \rho^{* 2}} .
$$

In figure $3 \tilde{G}(k)$ is shown for the first set of parameters of the SALR potential (see equation (10)), for the two extensions of $\tilde{u}^{* \mathrm{LR}}(r)$ for $r<1$. The second maximum for $r \approx 6$, present in experimental systems, occurs when equation (4) is used for $\tilde{u}^{* \operatorname{LR}}(r)$.

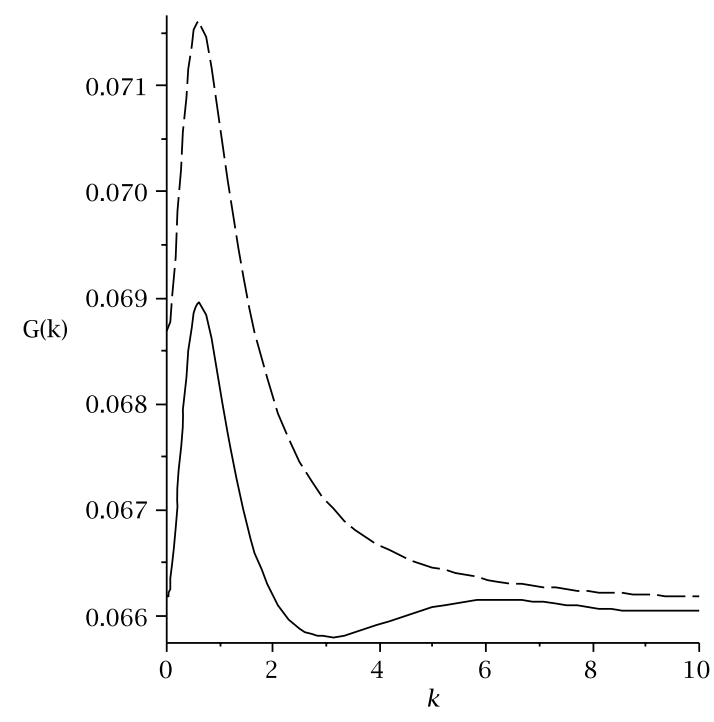

Figure 3. Correlation function in Fourier representation, $\tilde{G}(k)$ (equation (20)), in the diluted phase (structure factor is given by $S(k)=\tilde{G}(k) / \rho^{*}$ ) for $\mathcal{A}_{1}=1, \mathcal{A}_{2}=0.2, z_{1}=1$ and $z_{2}=0.5$ and $\rho^{*}=0.1$, and $T^{*}=1.58$. The dashed and solid lines are for $u^{\mathrm{LR}}=v$ and for $u^{\mathrm{LR}}$ given by equation (4) respectively.

The boundary of stability of $\Omega^{\mathrm{MF}}$ is given by

$$
\tilde{C}\left(k_{\mathrm{b}}\right)=0 .
$$

Note that the boundary of stability of the functional (12) significantly depends on the form of $\tilde{u}^{* \mathrm{LR}}(r)$ for $r<1$, where it is not uniquely defined. In the context of the mesoscopic theory, where $\tilde{u}^{* \mathrm{LR}}(r)$ in (12) should be replaced by $\tilde{u}^{* \mathrm{LR}}(r) g(r)$, the quantitative results depend on the assumed form of the correlation function $g(r)$ with density constrained to have a fixed form on the mesoscopic length scale. Here we assume $g(r)=\theta(r-1)$. The explicit expression for the boundary of stability of the uniform phase is

$$
\beta^{*}=\frac{(1+2 s)^{2}}{\rho^{*}(1-s)^{4}} .
$$

The MF line of instability depends on the state dependence of the temperature unit $\left|\tilde{u}^{\mathrm{LR}}\left(k_{\mathrm{b}}\right)\right|$. Instability with respect to separation of uniform phases occurs when $k_{\mathrm{b}}=0$, and equation (23) represents the spinodal line in this case. Metastable separation occurs provided that the condition (8) is satisfied, and is given by (23) with $\tilde{u}^{* \mathrm{LR}}\left(k_{\mathrm{b}}\right)$ replaced by $\tilde{u}^{* \mathrm{LR}}(0)$. 


\section{First order transition to microsegregated phases}

For weakly first-order transitions we assume that the density has the form

$$
\rho(\mathbf{x})=\rho_{0}^{*}+\eta(\mathbf{x}) .
$$

Next we expand $f_{\mathrm{h}}\left(\rho^{*}\right)$ about $\rho_{0}^{*}$, and we obtain

$$
\begin{aligned}
\beta \Delta \Omega^{\mathrm{MF}}\left[\rho_{0}^{*}, \eta(\mathbf{x})\right] & =\beta \Omega^{\mathrm{MF}}\left[\rho_{0}^{*}+\eta(\mathbf{x})\right]-\beta \Omega^{\mathrm{MF}}\left[\rho_{0}^{*}\right] \\
& =\frac{1}{2} \int_{\mathbf{k}} \tilde{\eta}(\mathbf{k}) \beta^{*} \tilde{u}^{* \mathrm{LR}}(k) \tilde{\eta}(-\mathbf{k})+\sum_{n=3}^{\infty} \frac{A_{n}}{n !} \int_{\mathbf{x}} \eta(\mathbf{x})^{n},
\end{aligned}
$$

where

$$
A_{n}=\left.\frac{\partial^{n} \beta f_{\mathrm{h}}(\rho)}{\partial \rho^{* n}}\right|_{\rho^{*}=\rho_{0}^{*}} .
$$

The stability requirement for $\eta \rightarrow \infty$ allows us for truncating the expansion at even $n \geqslant 4$. Here we shall limit ourselves to $n=4$. Explicit expressions for $A_{3}$ and $A_{4}$ are given in Appendix.

The functional (25) has a well know structure [23 26] considered before in phenomenological approaches to block copolymers, highly charged colloids and microemulsions. We repeat the same procedure and assume that $\eta(\mathbf{x})$ is periodic and can be written in the form

$$
\eta(\mathbf{x})=\sum_{n} \Phi_{n} g_{n}^{\mathrm{ph}}(\mathbf{x})
$$

where $g_{n}^{\mathrm{ph}}(\mathbf{x})$ represents orthonormal basis functions for the $n$-th shell that have the symmetry of the phase $p h$, and satisfy the normalization condition

$$
\frac{\int_{V_{u}} g_{n}(\mathbf{x})^{2}}{V_{u}}=1
$$

By $\int_{V_{u}}$ we denote an integration over the unit cell of the ordered structure. The volume of the unit cell is denoted by $V_{u}$. $\Phi_{n}$ is the $n$-th amplitude.

Stable or metastable phases correspond to the global or a local minimum of $\Delta \Omega^{\mathrm{MF}}\left[\rho_{0}^{*}, \eta(\mathbf{x})\right]$. At phase coexistence between two phases $\Delta \Omega^{\mathrm{MF}}\left[\rho_{0}^{*}, \eta(\mathbf{x})\right]$ for these phases takes equal values. At the transition between the fluid and the ordered phase $\Delta \Omega^{\mathrm{MF}}\left[\rho_{0}^{*}, \eta(\mathbf{x})\right]=0$.

\subsection{One-shell approximation}

Let us first limit ourselves to the one-shell approximation. For structures possessing different symmetries the Fourier transform of $g_{1}$ has the form

$$
\tilde{g}_{1}^{\mathrm{ph}}(\mathbf{k})=\frac{(2 \pi)^{d}}{\sqrt{2 n^{\mathrm{ph}}}} \sum_{j=1}^{n^{\mathrm{ph}}}\left(w \delta\left(\mathbf{k}-\mathbf{k}_{b}^{j}\right)+w^{*} \delta\left(\mathbf{k}+\mathbf{k}_{b}^{j}\right)\right)
$$

where $w w^{*}=1$ and $2 n^{\mathrm{ph}}$ is the number of vectors $\mathbf{k}_{b}^{j}$ in the first shell of the structure $p h$. For the first shell $\left|\mathbf{k}_{b}^{j}\right|=k_{\mathrm{b}}$. Since $u^{\mathrm{LR}}(k)$ assumes a minimum for $k=k_{\mathrm{b}}$, the contribution to the grand potential from the first shell is lower than the contributions from the remaining shells. The explicit forms of the functions $g_{1}^{\mathrm{ph}}$ in real-space representation for lamellar, hexagonal, bcc and gyroid phases are given in Appendix. Within the given symmetry, the amplitude $\Phi_{1}$ of the order parameter corresponds to the minimum $\Delta \Omega_{p h}^{\mathrm{MF}}$ of $\Delta \Omega^{\mathrm{MF}}\left[\rho_{0}^{*}+\Phi_{1} g_{1}^{\mathrm{ph}}\right]$ with respect to $\Phi_{1}$. As already shown by Leibler [24], for $A_{3} \neq 0$, the ordered phase, coexisting with the fluid, has the bcc symmetry in MF. For $A_{3}=0$ the transition is to the striped (lamellar) phase [24]. In the considered system $A_{3}\left(\rho^{*}\right)<0$ for $\rho^{*}<\rho_{\mathrm{c}}^{*}, A_{3}\left(\rho^{*}\right)=0$ for $\rho^{*}=\rho_{\mathrm{c}}^{*}$, and $A_{3}\left(\rho^{*}\right)>0$ for $\rho^{*}>\rho_{\mathrm{c}}^{*}$, where $\rho_{\mathrm{c}}^{*}$ 
is the critical density of the gas-liquid separation. In the PY approximation (14) $\rho_{\mathrm{c}}^{*} \approx 0.2457358$. From (25) - (28) we obtain in the one-shell approximation

$$
\Delta \Omega^{\mathrm{MF}}=\frac{1}{2} \tilde{C}\left(k_{\mathrm{b}}\right) \Phi_{1}^{2}+\frac{A_{3} \kappa_{3}^{\mathrm{ph}}}{3 !} \Phi_{1}^{3}+\frac{A_{4} \kappa_{4}^{\mathrm{ph}}}{4 !} \Phi_{1}^{4}
$$

where

$$
\kappa_{n}^{\mathrm{ph}}=\frac{\int_{V_{u}} g_{1}^{\mathrm{ph}}(\mathbf{x})^{n}}{V_{u}}
$$

From the extremum condition

$$
\frac{\partial \Delta \Omega^{\mathrm{MF}}}{\partial \Phi_{1}}=\tilde{C}\left(k_{\mathrm{b}}\right) \Phi_{1}+\frac{A_{3} \kappa_{3}^{\mathrm{ph}}}{2} \Phi_{1}^{2}+\frac{A_{4} \kappa_{4}^{\mathrm{ph}}}{3 !} \Phi_{1}^{3}=0
$$

we obtain the amplitude

$$
\Phi_{1+,-}=\frac{\sqrt{3}\left[-\sqrt{3} A_{3} \kappa_{3} \pm \sqrt{3\left(A_{3} \kappa_{3}\right)^{2}-8 A_{4} \kappa_{4} \tilde{C}\left(k_{\mathrm{b}}\right)}\right]}{2 A_{4} \kappa_{4}}
$$

for each phase characterized by different geometric factors $\kappa_{n}$ given in Appendix. Transition between the disordered and the bcc phase, as well as the amplitude of the density profiles along the transition line are given by analytic expressions in [17], where the remaining transition lines between ordered phases, obtained numerically, are also shown.

\subsection{Two-shell approximation}

The accuracy of the one-shell approximation depends on the shape of $\tilde{u}^{\mathrm{LR}}(k)$, in particular, on the ratio $\tilde{u}^{\mathrm{LR}}\left(k_{b 2}\right) / \tilde{u}^{\mathrm{LR}}\left(k_{\mathrm{b}}\right)$, where $k_{b 2}$ is the wavenumber corresponding to the second shell. The approximation is the worse the smaller is the above ratio, since the second shell should not be disregarded when it leads to comparable energy as the first shell. For a given shape of the potential, the value of $k_{b 2}$ matters, and it is different for different structures. As discussed in detail in [17, 27], for the gyroid phase $k_{b 2}=k_{\mathrm{b}} 2 / \sqrt{3}$, whereas for the remaining phases $k_{b 2} / k_{\mathrm{b}}$ is much larger, so the value of $\tilde{u}^{\mathrm{LR}}\left(k_{b 2}\right) / \tilde{u}^{\mathrm{LR}}\left(k_{\mathrm{b}}\right)$ is much larger too. We thus consider the gyroid phase in the two-shell approximation, and the remaining phases in the one-shell approximation. In the two-shell approximation the grand potential assumes the form

$$
\begin{aligned}
\Delta \Omega^{\mathrm{MF}}= & \frac{1}{2} \tilde{C}\left(k_{\mathrm{b}}\right) \Phi_{1}^{2}+\kappa_{3} \frac{A_{3}}{3 !} \Phi_{1}^{3}+\kappa_{4} \frac{A_{4}}{4 !} \Phi_{1}^{4} \\
& +\frac{1}{2} \tilde{C}\left(k_{2 b}\right) \Phi_{2}^{2}+\kappa_{0,3} \frac{A_{3}}{3 !} \Phi_{2}^{3}+\kappa_{0,4} \frac{A_{4}}{4 !} \Phi_{2}^{4}+\kappa_{2,1} \frac{A_{3}}{2} \Phi_{1}^{2} \Phi_{2}+\kappa_{2,2} \frac{A_{4}}{2 ! 2 !} \Phi_{1}^{2} \Phi_{2}^{2},
\end{aligned}
$$

where the geometric factors

$$
\kappa_{n, m}^{\mathrm{ph}}=\frac{\int_{V_{u}} g_{1}^{\mathrm{ph}}(\mathbf{x})^{n} g_{2}^{\mathrm{ph}}(\mathbf{x})^{m}}{V_{u}} .
$$

for the gyroid phase are given in Appendix. The minimum condition with respect to $\Phi_{i}$ gives

$$
\begin{aligned}
\Phi_{1 \pm} & = \pm \sqrt{-\frac{\tilde{C}\left(k_{b 2}\right) \Phi_{2}+\kappa_{0,3} \frac{A_{3}}{2} \Phi_{2}^{2}+\kappa_{0,4} \frac{A_{4}}{3 !} \Phi_{2}^{3}}{\kappa_{2,1} \frac{A_{3}}{2}+\kappa_{2,2} \frac{A_{4}}{2} \Phi_{2}}}, \\
\Phi_{2 \pm} & =\frac{-\kappa_{2,1} A_{3} \pm \sqrt{\Delta}}{\kappa_{2,2} A_{4}}
\end{aligned}
$$

with

$$
\Delta=\kappa_{2,1}^{2} A_{3}^{2}-2 \kappa_{2,2} A_{4}\left(\tilde{C}\left(k_{\mathrm{b}}\right)+\kappa_{3,0} \frac{A_{3}}{2} \Phi_{1}+\kappa_{4,0} \frac{A_{4}}{6} \Phi_{1}^{2}\right)
$$


For $\rho^{*}<\rho_{\mathrm{c}}^{*}$ and $\rho^{*}>\rho_{\mathrm{c}}^{*}$ the minimum of $\Delta \Omega^{\mathrm{MF}}$ corresponds to $\Phi_{1+}$ and $\Phi_{2-}$, and to $\Phi_{1-}$ and $\Phi_{2+}$ respectively. It is important to note that the structure given by two-shell and by one-shell approximation is significantly different. Namely, in the one-shell approximation a single network of excess density is present, whereas for $\Phi_{1} \Phi_{2}<0$ two much thinner networks of excess density are formed. The phase diagrams calculated for the two potentials with parameters given in equation (10) (see figures 1,2) are shown in figures 4, 5, Note that despite significantly different shape

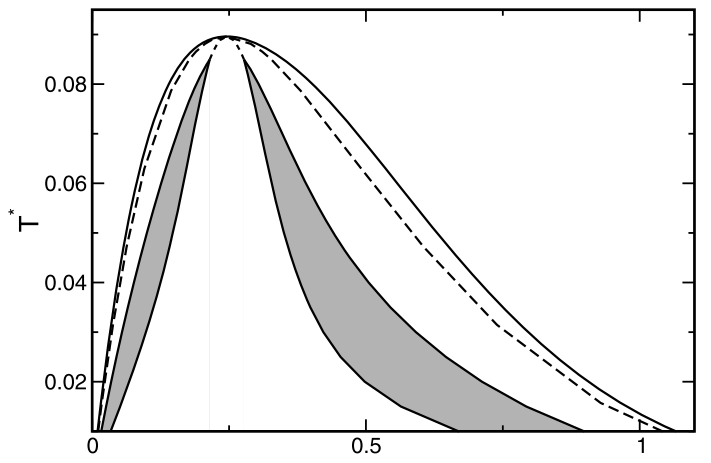

Figure 4. Phase diagram obtained in MF approximation with the bcc, hexagonal and lamellar phases considered in the one-shell and the gyroid phase in the two-shell approximation for $\mathcal{A}_{1}=1, \mathcal{A}_{2}=0.2, z_{1}=1$ and $z_{2}=0.5$. The outer solid lines are the coexistence lines between the disordered and the bcc crystal phases, the bcc and the hexagonal phases coexist along the dashed lines, along the dotted lines the hexagonal and the lamellar phases coexist, and inside the shaded regions the gyroid phase is stable. For densities lower than $\rho_{\mathrm{c}}^{*}$ clusters of particles form ordered patterns, whereas for $\rho^{*}>\rho_{\mathrm{c}}^{*}$ the pattern is formed by bubbles - regions with depleted particle density.

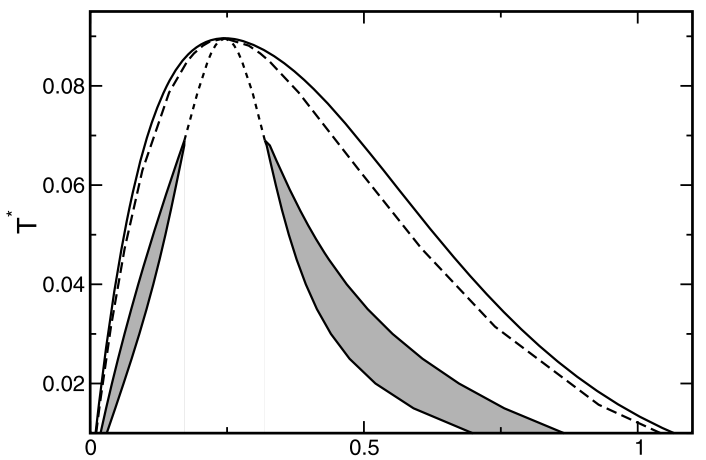

Figure 5. Phase diagram obtained in MF approximation with the bcc, hexagonal and lamellar phases considered in one-shell and the gyroid phase in the two-shell approximation for $\mathcal{A}_{1}=1$, $\mathcal{A}_{2}=0.05, z_{1}=3$ and $z_{2}=0.5$. The outer solid lines are the coexistence lines between the disordered and the bcc crystal phases, the bcc and the hexagonal phases coexist along the dashed lines, along the dotted lines the hexagonal and the lamellar phases coexist, and inside the shaded regions the gyroid phase is stable. For densities lower than $\rho_{\mathrm{c}}^{*}$ clusters of particles form ordered patterns, whereas for $\rho^{*}>\rho_{\mathrm{c}}^{*}$ the pattern is formed by bubbles - regions with depleted particle density.

of the interaction potential, the extent of the stability region of the gyroid phase on the phase diagram given in reduced variables $\left(\rho^{*}, T^{*}\right)$ is similar. Moreover, the phase diagram in variables $\left(\rho^{*}, T^{*}\right)$ is similar to the phase diagram obtained earlier [17] for the SALR potential characterized by the parameters $A_{1}=140 \exp (8.4), A_{2}=30 \exp (1.55), z_{1}=8.4$ and $z_{2}=1.55$, and corresponding to $k_{\mathrm{b}} \approx 1.94026$. The ratio of $\tilde{v}\left({ }_{b 2}\right) / \tilde{v}\left(k_{\mathrm{b}}\right)$ for the cases I and II and for the previously considered potential takes the values $0.9762993733,0.8973863512,0.9119505483$ respectively. In the 
case of large clusters (case I, $\pi / k_{\mathrm{b}} \approx 5$ ) the gyroid phase extents to higher temperatures and occupies a larger range of densities than in the case of small clusters (case II, $\pi / k_{\mathrm{b}} \approx 1.7$ and [17], $\left.\pi / k_{\mathrm{b}} \approx 1.6\right)$. We conclude that in the simple MF approximation, the phase diagram in reduced variables, although not universal beyond the one-shell approximation, rather weakly depends on the shape of the effective interaction potential. The region of stability of the gyroid phase increases when the minimum of the interaction potential in Fourier representation becomes shallower, i.e., when $\tilde{v}\left(k_{b 2}\right) / \tilde{v}\left(k_{b}\right)$ increases.

\section{Summary}

We have considered the SALR potential representing a wide class of biological and soft matter systems in MF approximation and in the case of weak ordering. We first discussed the properties of such kind of potential, and next focused on determination of the phase diagram in reduced variables. Our main goal was to determine the stability region of gyroid phase with Ia3d symmetry for various shapes of the SALR potential. We have chosen two qualitatively different cases, I and II, corresponding to very large clusters and to small clusters of colloids, respectively. We have found that the phase diagrams in reduced units in these two cases are similar. The gyroid phase is located between the hexagonal and the lamellar phase, and may be related to a branched network of clusters observed experimentally and in simulations [6, 7, 14]. The smaller is the difference between the energy gain associated with the formation of a planar density wave with the wave number $k \approx k_{\mathrm{b}}$ and the gain for the most probable wavelength $k=k_{\mathrm{b}}$, the more favorable is the gyroid phase.

We expect that the free-energy landscape contains many local minima corresponding to a distorted network, and that the formation of thermodynamically stable regular structure of the gyroid phase in experimental system may be preempted by a glass-like disordered branched network that locally resembles the gyroid phase. Confirmation of the existence of the predicted gyroid phase by experiment or simulation remains a challenge.

\section{Acknowledgement}

This work is dedicated to Prof. Ihor Mryglod on the occasion of his 50 birthday. We gratefully acknowledge partial support by the Polish Ministry of Science and Higher Education, Grant No. NN 202006034.

\section{Appendix. Explicit expressions of functions and parameters}

Parameters in the functional (25)

$$
\begin{aligned}
& A_{3}(\rho)=\frac{12 s^{3}+20 s^{2}+5 s-1}{\rho^{2}(1-s)^{5}} \\
& A_{4}(\rho)=\frac{2-12 s+30 s^{2}+112 s^{3}+48 s^{4}}{\rho^{3}(1-s)^{6}}
\end{aligned}
$$

geometric factors in equations (30) and (34)

$$
\begin{aligned}
\kappa_{3}^{\mathrm{bcc}} & =\sqrt{\frac{4}{3}}, \\
\kappa_{4}^{\mathrm{bcc}} & =\frac{15}{4},
\end{aligned}
$$




$$
\begin{array}{r}
\kappa_{3,0}^{\mathrm{Ia} 3 \mathrm{~d}}=\frac{1}{\sqrt{6}}, \\
\kappa_{4,0}^{\mathrm{Ia} 3 \mathrm{~d}}=\frac{17}{8}, \\
\kappa_{0,3}^{\mathrm{Ia} 3 \mathrm{~d}}=\frac{2}{\sqrt{3}}, \\
\kappa_{0,4}^{\text {Ia3d }}=\frac{15}{4}, \\
\kappa_{2,1}^{\text {Ia3d }}=-\frac{\sqrt{3}}{6}, \\
\kappa_{2,2}^{\text {Ia3d }}=\frac{1}{2}, \\
\kappa_{3}^{\text {hex }}=\sqrt{\frac{2}{3}}, \\
\kappa_{4}^{\text {hex }}=\frac{5}{2}, \\
\kappa_{3}^{\text {lam }}=0, \\
\kappa_{4}^{\text {lam }}=\frac{3}{2},
\end{array}
$$

where $\kappa_{n, 0} \equiv \kappa_{n}$

Expressions for the functions $g_{1}(\mathbf{x})$. We use the notation $\mathbf{x}=\left(x_{1}, x_{2}, x_{3}\right)$.

$$
\begin{aligned}
g_{1}^{\ell}(\mathbf{x})= & \sqrt{2} \cos \left(k_{\mathrm{b}} x_{1}\right) \\
g_{1}^{\text {hex }}(\mathbf{x})= & \sqrt{\frac{2}{3}}\left[\cos \left(k_{\mathrm{b}} x_{1}\right)+2 \cos \left(\frac{k_{\mathrm{b}} x_{1}}{2}\right) \cos \left(\frac{\sqrt{3} k_{\mathrm{b}} x_{2}}{2}\right)\right] \\
g_{1}^{\mathrm{bcc}}(\mathbf{x})= & \frac{1}{\sqrt{3}} \sum_{i<j}\left(\cos \left(\frac{k_{\mathrm{b}}\left(x_{i}+x_{j}\right)}{\sqrt{2}}\right)+\cos \left(\frac{k_{\mathrm{b}}\left(x_{i}-x_{j}\right)}{\sqrt{2}}\right)\right) \\
g_{1}^{\text {Ia } 3 \mathrm{~d}}(\mathbf{x})= & \sqrt{\frac{8}{3}}\left[\cos \left(\frac{k_{\mathrm{b}} x_{1}}{\sqrt{6}}\right) \sin \left(\frac{k_{\mathrm{b}} x_{2}}{\sqrt{6}}\right) \sin \left(\frac{2 k_{\mathrm{b}} x_{3}}{\sqrt{6}}\right)+\cos \left(\frac{k_{\mathrm{b}} x_{2}}{\sqrt{6}}\right) \sin \left(\frac{k_{\mathrm{b}} x_{3}}{\sqrt{6}}\right) \sin \left(\frac{2 k_{\mathrm{b}} x_{1}}{\sqrt{6}}\right)\right. \\
& \left.+\cos \left(\frac{k_{\mathrm{b}} x_{3}}{\sqrt{6}}\right) \sin \left(\frac{k_{\mathrm{b}} x_{1}}{\sqrt{6}}\right) \sin \left(\frac{2 k_{\mathrm{b}} x_{2}}{\sqrt{6}}\right)\right]
\end{aligned}
$$

Expression for the function $g_{2}(\mathbf{x})$ for the gyroid phase

$$
g_{2}^{\mathrm{Ia} 3 \mathrm{~d}}(\mathbf{x})=\frac{1}{\sqrt{3}} \sum_{i<j}\left(\cos \left(\frac{2 k_{\mathrm{b}}\left(x_{i}+x_{j}\right)}{\sqrt{6}}\right)+\cos \left(\frac{2 k_{\mathrm{b}}\left(x_{i}-x_{j}\right)}{\sqrt{6}}\right)\right) .
$$

\section{References}

1. Sear R.P., Gelbart W.M., J. Chem. Phys., 1999, 110, 4582.

2. Sciortino F., Mossa S., Zaccarelli E., Tartaglia P., Phys. Rev. Lett., 2004, 93, 055701.

3. Stradner A., Sedgwick H., Cardinaux F., Poon W.C.K., Egelhaaf S.U., Schurtenberger P., Nature, 2004, 432, 492.

4. Sedgwick H., Egelhaaf S., Poon W., J. Phys. Condens. Matter, 2004, 16, S4913.

5. Imperio A., Reatto L., J. Phys.: Cond. Mat., 2004, 16, 3769.

6. Sciortino F., Tartaglia P., Zaccarelli E., J. Phys. Chem. B, 2005, 109, 21942.

7. Campbell A.I., J.Anderson V., van Duijneveldt J.S., Bartlett P., Phys. Rev. Lett., 2005, 94, 208301.

8. Pini D., Parola A., Reatto L., J. Phys.: Cond. Mat., 2006, 18, S2305.

9. de Candia A., Del Gado E., Fierro A., Sator N., Tarzia M., Coniglio A., Phys. Rev. E, 2006, 74, 010403(R). 
10. Archer A.J., Pini D., Evans R., Reatto L., J. Chem. Phys., 2007, 126, 014104.

11. Archer A.J., Wilding N.B., Phys. Rev. E, 2007, 76, 031501.

12. Archer A.J., Phys. Rev. E, 2008, 78, 031402.

13. Archer A., Ionescu, Pini D., Reatto L., J. Phys.: Cond. Mat., 2008, 20, 415106.

14. Toledano J.C.F., Sciortino F., Zaccarelli E., Soft Matter, 2009, 5, 2390.

15. Sennato S., Truzzolillo D., Bordi F., Sciortino F., Cametti C., Colloid Surface, 2009, 343, 34.

16. Shukla A., Mylonas E., Di Cola E., Finet S., Timmins P., Narayanan T., Svergun D.I., PNAS, 2008, 105, 5075.

17. Ciach A., Phys. Rev. E, 2008, 78, 061505.

18. Ciach A., AIP Conference Proceedings, 2009, 1198, 13.

19. Patsahan O., Condens. Matter Phys., 2004, 7, 35.

20. Patsahan O., Mryglod I., J. Phys.: Cond. Mat., 2004, 16, L235.

21. Patsahan O., Mryglod I., Condens. Matter Phys., 2004, 7, 755.

22. Patsahan O., Patsahan T., AIP Conference Proceedings, 2009, 1198, 124.

23. Brazovskii S.A., Sov. Phys. JETP, 1975, 41, 8.

24. Leibler L., Macromolecules, 1980, 13, 1602.

25. Ciach A., Góźdź W.T., Stell G., J. Phys. Cond. Mat., 2006, 18, 1629.

26. Ciach A., Góźdź W.T., Annu. Rep. Prog. Chem. C, 2001, 97, 269, and references therein.

27. Podneks V.E., Hamley I.W., Pis'ma Zh. Exp. Teor. Fiz., 1996, 64, 564.

\title{
Мезоскопічний опис мережо-формуючих кластерів слабо заряджених колоїдів
}

\author{
А. Цях., В.Т. Гузьдзь \\ Інститут фізичної хімії Польської Академії Наук, Варшава, Республіка Польща
}

В рамках мезоскопічної польової теорії вивчаються системи, що складаються із сферичних заряджених частинок у розчинниках, які містять контріони та індукують короткосяжне притягання. Ми обмежуємося наближенням середнього поля (СП) і слабким впорядкуванням. Ми обговорюємо властивості потенціалів, що складаються із сильного короткосяжного притягання і слабкого далекосяжного відштовхування (SALR) у контексті формування неоднорідного розподілу частинок на мезоскопічних масштабах довжин замість макроскопічного фазового відокремлення. У попередній роботі показано, що формуються сферичні, циліндричні і щілиноподібні кластери частинок, і для достатньо низьких температур ці кластери формують впорядковані періодичні об'ємоцентричні, гексагональні і ламеларні фази. Крім того, було передбачено гіроїдну фазу, в якій формуються два переплетені регулярні мережоподібні кластери, що розгалужуються в потрійному з'єднанні. Було знайдено, що при відповідному масштабуванні густини і температури, лінії співіснування між різними впорядкованими фазами є універсальними в наближенні СП, за винятком гіроїдної фази. У цій статті визначається фазова діаграма для двох виборів потенціалу SALR: такого, що відповідає великій області притягуючої частини потенціалу, та такого, що відповідає малій області притягання. Ми виявляємо, що область стабільності гіроїдної фази дуже слабо залежить від форми потенціалу SALR у межах наближеної теорії.

Ключові слова: колоїди, кластери, самоорганізація, фазові переходи лад-безлад 